\title{
Protected Long-Distance Guiding of Hypersound Underneath a Nanocorrugated Surface
}

\author{
Dmytro D. Yaremkevich, Alexey V. Scherbakov,* Serhii M. Kukhtaruk, Tetiana L. Linnik, \\ Nikolay E. Khokhlov, Felix Godejohann, Olga A. Dyatlova, Achim Nadzeyka, Debi P. Pattnaik, \\ Mu Wang, Syamashree Roy, Richard P. Campion, Andrew W. Rushforth, Vitalyi E. Gusev, \\ Andrey V. Akimov, and Manfred Bayer
}

Cite This: https://dx.doi.org/10.1021/acsnano.0c09475

Read Online

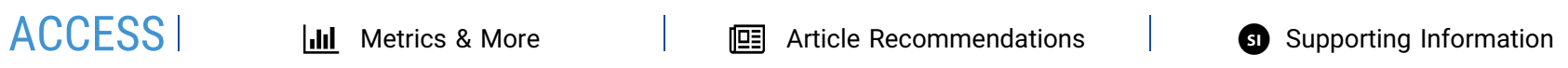

ABSTRACT: In nanoscale communications, high-frequency surface acoustic waves are becoming effective data carriers and encoders. On-chip communications require acoustic wave propagation along nanocorrugated surfaces which strongly scatter traditional Rayleigh waves. Here, we propose the delivery of information using subsurface acoustic waves with hypersound frequencies of $\sim 20 \mathrm{GHz}$, which is a nanoscale analogue of subsurface sound waves in the ocean. A bunch of subsurface hypersound modes are generated by pulsed optical excitation in a multilayer semiconductor structure with a metallic nanograting on top. The guided hypersound modes propagate coherently beneath the nanograting, retaining the

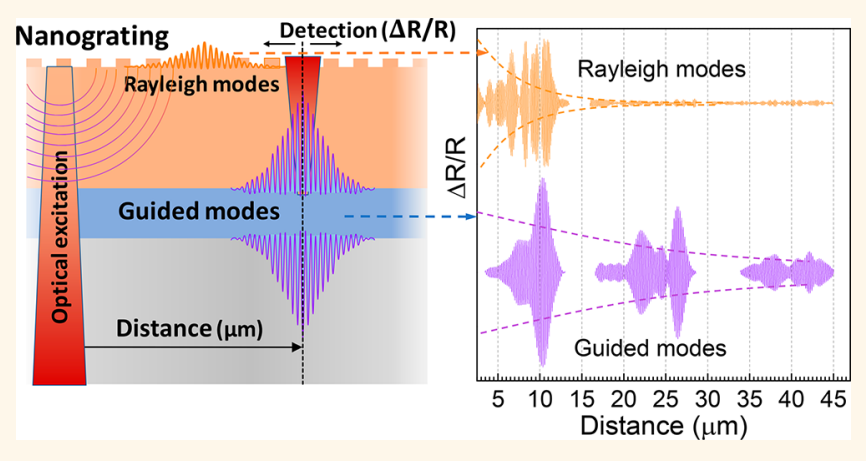
surface imprinted information, at a distance of more than $50 \mu \mathrm{m}$ which essentially exceeds the propagation length of Rayleigh waves. The concept is suitable for interfacing single photon emitters, such as buried quantum dots, carrying coherent spin excitations in magnonic devices and encoding the signals for optical communications at the nanoscale.

KEYWORDS: coherent phonons, surface acoustic waves, acoustic waveguiding, nanogratings, superlattices, pump-probe spectroscopy

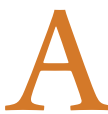

coustic waves with terahertz $(\mathrm{THz})$ and gigahertz $(\mathrm{GHz})$ frequencies, which are often referred to as hypersound, are attractive for applications in quantum computing, sensing, and communications. ${ }^{1-4} \mathrm{~A}$ major advantage is the nanometer wavelength of hypersound which, in contrast to photons, is comparable with the size of singleelectron and single-photon devices. Surface acoustic waves (SAWs) of hypersound frequencies, being the high-frequency counterpart of traditional MHz SAWs, have been implemented successfully in quantum technologies as an instrument to control single electron, ${ }^{5,6}$ photonic, ${ }^{7,8}$ and spintronic ${ }^{9-11}$ devices. It has been proposed to use hypersound to connect different nanoobjects (e.g., qubits) on-chip in analogy with wires connecting the elements of traditional electronics. ${ }^{12-15}$ For this, the propagation distance of the acoustic signal should be at least comparable with the distance between the source and receiver. SAWs with frequencies up to $50 \mathrm{GHz}$ can propagate on flat surfaces over millimeter distances, ${ }^{16}$ but nanodevice architectures on single chips inevitably require nanometer processing and surface corrugation upon which the hypersound diffracts, reducing the SAW's mean free path from millimeters to micrometers. ${ }^{17}$

In this article, we demonstrate experimentally how subsurface hypersound propagates from the source to the receiver on distances more than $50 \mu \mathrm{m}$ underneath a nanopatterned metallic layer. The principle is schematically demonstrated in Figure 1a where surface and subsurface waves are propagating along a nanostructured surface. Both waves are generated at point $\mathrm{A}$ and serve to deliver the information, for example, a spatially or temporally modulated signal, to point C. Traditional SAWs, that is, Rayleigh-like waves, do not reach the destination due to diffraction and scattering at the

Received: November 11, 2020

Accepted: January 29, 2021 


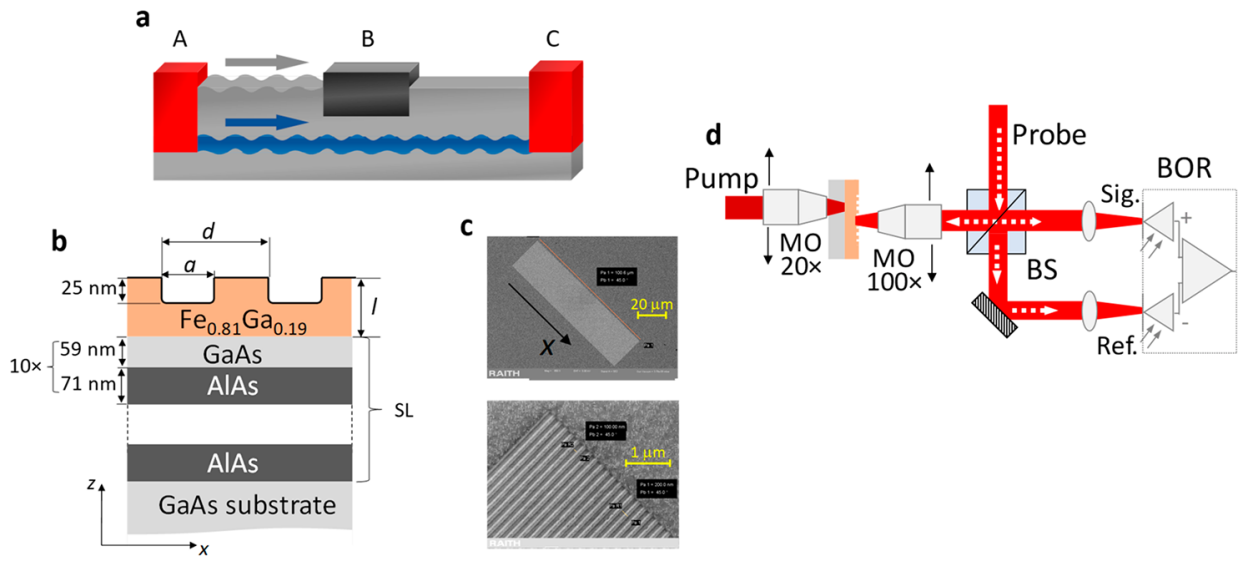

Figure 1. Experimental setup. (a) Basic concept: Surface waves traveling from $A$ to $C$ are scattered by object B, while subsurface waves freely propagate beneath the corrugated surface. (b) Scheme of the sample. (c) SEM images of the nanograting. (d) Schematic of the experimental setup for measuring phonon propagation with the pump and probe spots separated in space.
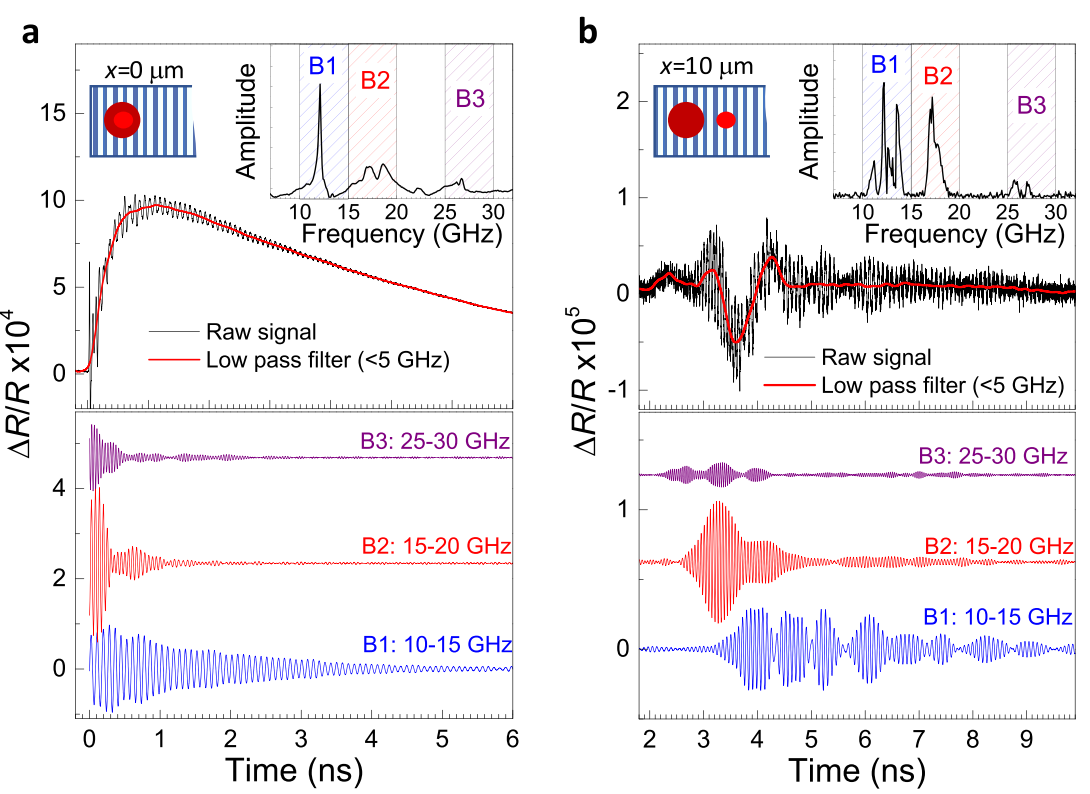

Figure 2. Experimental signals. (a) Upper panel: Signal detected when the pump and probe spots overlap in space; the inset is the FFT of the temporal signal. Lower panel: Temporal traces after filtering of the measured signal from the upper panel in three frequency bands marked in the inset. (b) Same as (a) but measured when the pump and probe spots are separated on the surface by $10 \mu \mathrm{m}$.

nanoobject B located on the SAW's path. ${ }^{18}$ Alternatively, subsurface waves continue to propagate under object B and do not feel any surface corrugation, similar to how a submarine does not feel the storm at the sea surface. This idea is a nanometer analogue for the delivery of acoustic signals using nonattenuating guided underwater sound waves. ${ }^{19}$ To realize this concept in practice, the subsurface hypersound should propagate parallel to the surface with preserved coherence and without strong attenuation and leakage to the bulk. Reaching this goal requires the design of structures that support long propagating hypersound modes and the development of methods to generate and detect such modes. In the present work, we achieve both goals. We demonstrate experimentally the propagation of coherent hypersound wavepackets, with a central frequency of $\sim 20 \mathrm{GHz}$, on macroscopic distances which essentially exceed the mean free path of high-frequency Rayleigh waves at the corrugated surface.

\section{RESULTS AND DISCUSSION}

The samples consist of lateral one-dimensional nanogratings (NGs) fabricated from metallic $\left(\mathrm{Fe}_{0.81} \mathrm{Ga}_{0.19}\right)$ layers deposited on GaAs substrates. Two samples were studied in our experiment. In the first sample (see Figure 1b), the metallic layer with a thickness of $l=105 \mathrm{~nm}$ was deposited onto a GaAs/AlAs superlattice (SL) grown on a (001)-GaAs substrate. As we shall show, the elastic parameters of the SL allow the waveguiding effect for hypersound. In the second sample, the metallic layer with $l=100 \mathrm{~nm}$ was deposited directly onto a GaAs substrate without a SL. The NGs with a lateral size of $25 \times 100 \mu \mathrm{m}^{2}$ consist of grooves of $25 \mathrm{~nm}$ depth milled parallel to the [010] crystallographic direction of the GaAs substrate. The grooves and stripes have the same width $a$, and the corresponding period $d$ in the two gratings has values $d=200$ and $150 \mathrm{~nm}$, respectively. The scanning electron microscope image of the NG with $d=200 \mathrm{~nm}$ is shown in Figure 1c. 

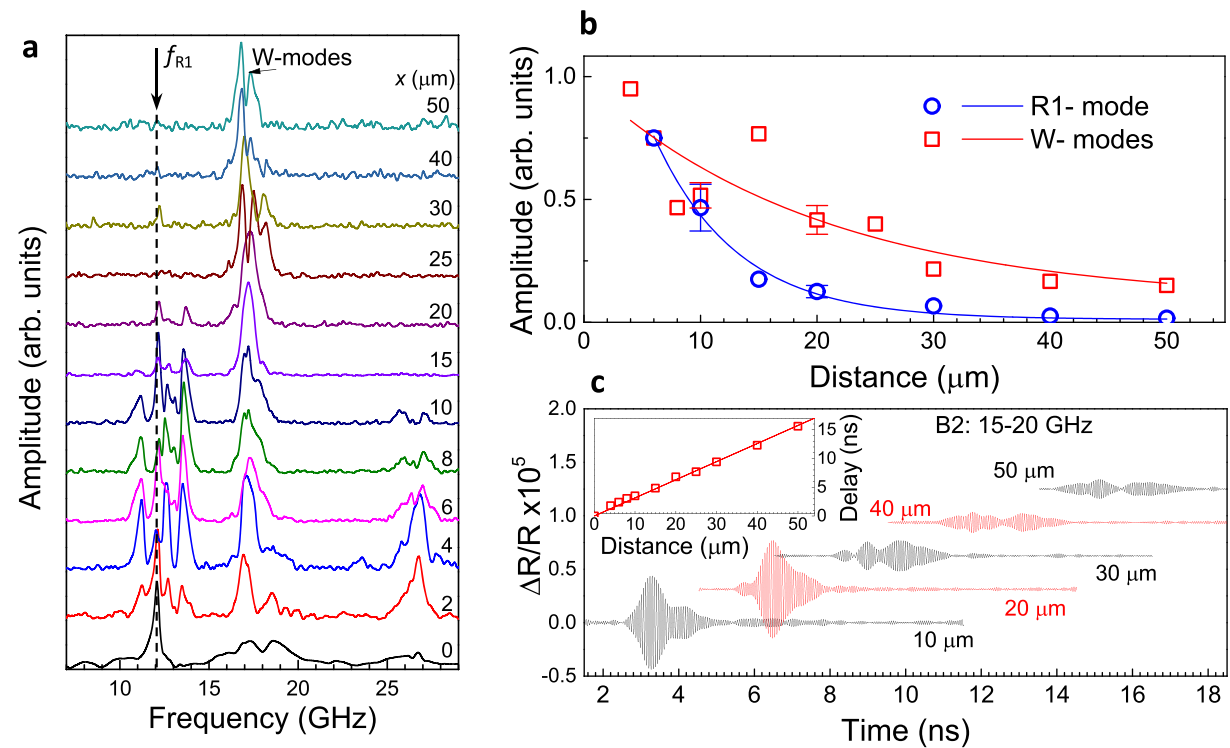

Figure 3. Dependence on the distance between source and detector. (a) FFTs obtained from the signals measured at various distances $x$ between the pump and probe spots. Each spectrum is normalized to its maximum spectral amplitude. (b) Dependences of the spectral amplitude of the filtered signals for the Rayleigh wave (R1 mode) and the mean spectral density for the waveguide modes (W-modes) on the distance between the pump and probe spots. Solid lines show the fits of the experimental data by an exponential decay, which serve to estimate the averaged mean free path for the propagating modes. The data and fit curves are normalized for clear presentation. (c) Temporal signals after filtering in the frequency band $\mathrm{B} 2$ for various distances between the pump and probe spots; the inset shows the dependence of the arrival time of the bunch around $17 \mathrm{GHz}$ on the distance.

Hypersound waves are excited and detected optically using a picosecond ultrasonic pump-probe technique. ${ }^{20,21}$ The schematic of the experiment is shown in Figure 1d. The pump beam from the femtosecond laser is sent in from the side of the GaAs substrate, which is transparent for the laser wavelength $1050 \mathrm{~nm}$, and focused onto the metallic film on a Gaussian spot with radius $\sigma=1 \mu \mathrm{m}$. The probe pulses from another laser with wavelength $780 \mathrm{~nm}$ are focused on a spot with a radius of $0.5 \mu \mathrm{m}$ on the metallic grating. The femtosecond pump pulse induces instantaneously thermal stress in the metallic layer, leading to the generation of the acoustic wavepacket with hypersound frequencies up to 100 $\mathrm{GHz}{ }^{20}$ In the presence of the NG, the generated hypersound propagates along the $x$-direction parallel to the surface and has wavevector components:

$$
q_{x}=q_{\mathrm{P}}+n(2 \pi / d) \quad n=0,1,2, \ldots
$$

where $x$ is the [100] direction perpendicular to the grooves (see Figure $1 \mathrm{~b}$ ), and $q_{\mathrm{P}} \ll 2 \pi / d$ is the wavevector limited by the size of the pump laser spot (see Methods). The detected reflectivity probe signal $\Delta R(t)$ is sensitive to waves with the same $q_{x}$ given by eq 1 . In the present experiments, we concentrate on measurements of the probe signals on distances up to $80 \mu \mathrm{m}$ from the pump spot along the $x$-axis $(x=0$ corresponds to the center of the pump spot, $t=0$ corresponds to the time moment when the pump pulse hits the studied structure).

The measured signal $\Delta R(t)$ at $x=0$, that is, at the point of spatial overlap of the pump and probe spots, in the grating with $d=200 \mathrm{~nm}$ and the GaAs/AlAs SL subsurface layer is shown in the upper panel of Figure 2a. The signal shows highfrequency oscillations sitting on a slowly varying background. The origin of the oscillations is elastic vibrations, that is, coherent hypersound waves, while the slow background, shown by the red solid line, is known to be due to the modulation of the refractive index by hot electrons, thermal phonons, and low-frequency acoustic motion of the surface excited by the optical pump pulse. $^{20,21}$ The inset in Figure 2a shows the fast Fourier transform (FFT) of the measured signal within three spectral bands: B1 $(10-15 \mathrm{GHz}), \mathrm{B} 2(15-20 \mathrm{GHz})$, and B3 $(25-30 \mathrm{GHz})$. The low-frequency band $\mathrm{B} 1$ includes an intense narrow spectral line at $f_{\mathrm{R} 1}=12.1 \mathrm{GHz}$ which corresponds to the first-order Rayleigh-like mode observed earlier in experiments with NGs. ${ }^{2-26}$ The other spectral bands include several surface modes, quasi-surface (skimming) modes, and bulk modes, which are commonly seen in experiments with gratings when the pump and probe pulses overlap. ${ }^{17,25,26}$ The lower panel shows the temporal signals after filtering in the different spectral bands. The signal in B1 has the longest lifetime, while the oscillations in the bands B2 and B3 decay much more quickly.

Figure $2 \mathrm{~b}$ shows the results for $\Delta R(t)$ measured when the pump and probe spots are separated in space by $10 \mu \mathrm{m}$ along the $x$-direction. In this case, only hypersound waves propagating along the surface perpendicular to the grooves are detected. From the raw signal (Figure 2a), it is seen that the slowly varying background is absent, which is explained by localization of thermal phonons and hot electrons inside the pump spot. The measured signals $\Delta R(t)$ show oscillations that sit on a background from the pulse with a duration of $\sim 1 \mathrm{~ns}$ (shown by the solid red line). This nanosecond pulse corresponds to the generated acoustic signal with $q_{x} \leq q_{\mathrm{P}}$ (n $=0),{ }^{27}$ which is excited also in a plain film without $\mathrm{NG}$, and will not be discussed further. The filtered signals (lower panel) and their FFTs (inset) differ significantly from the case $x=0$ : There are several hypersound modes in $\mathrm{B} 1$, and the spectral line in $\mathrm{B} 2$ centered at $f_{\mathrm{W}} \sim 17 \mathrm{GHz}$ has an intensity comparable with the modes in B1. There is a weak contribution from high-frequency modes in B3. The signals in the different spectral bands appear at different delay times $t_{i}$ 

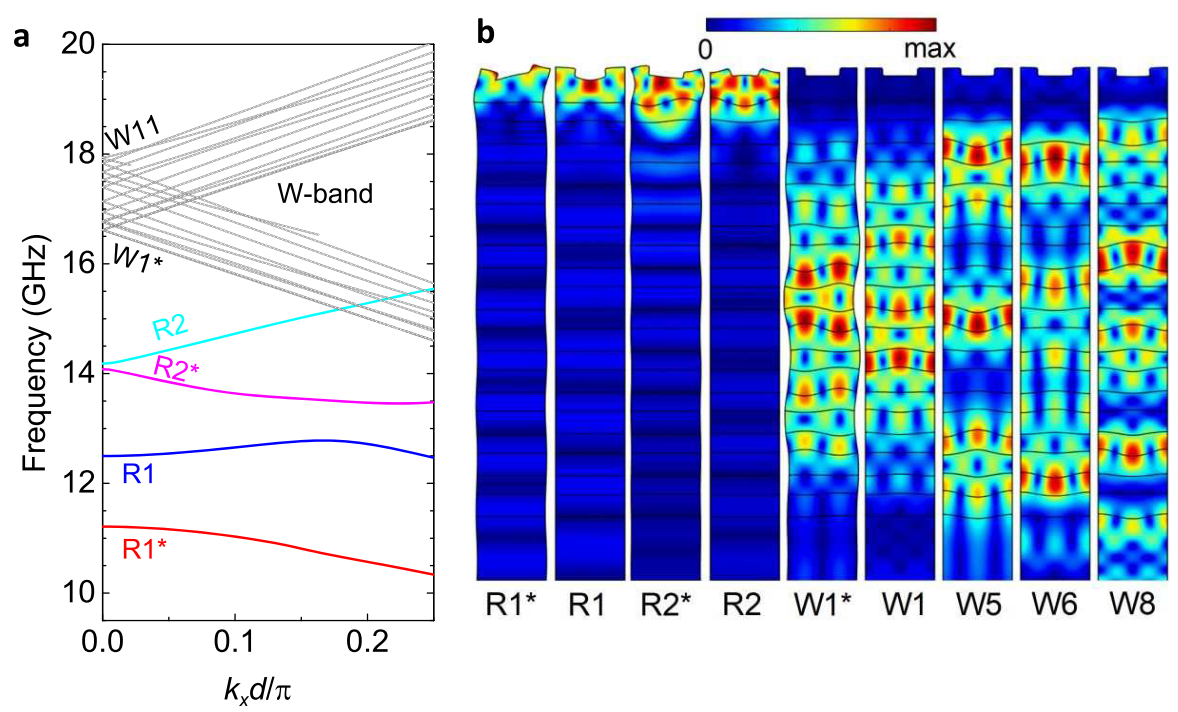

Figure 4. Dispersions and spatial distributions of the hypersound modes. (a) Calculated dispersion curves for hypersound modes propagating along the surface in the structure with the $\mathrm{Fe}_{0.81} \mathrm{Ga}_{0.19}$ nanograting with $d=200 \mathrm{~nm}$ deposited on the GaAs/AlAs SL. (b) Spatial distribution of the absolute value of displacement vector (exaggerated for clarity) for the Rayleigh waves (R1 and R1*) localized in the metallic layer with the nanograting and for five waveguiding (W) phonon modes localized in the GaAs/AlAs SL.

$\leq x / s_{i}$ (s is the group velocity of the wavepacket in the corresponding frequency band) relative to the pump pulse, which suggests different velocities $s_{i}$ of the corresponding hypersound modes. All reflectivity traces show beatings in the filtered signals which point toward multiple mode composition of the hypersound waves in each spectral band.

Figure 3 a shows the evolution of the FFTs with the increase of the distance to $x=50 \mu \mathrm{m}$. The normalized spectra show that only the hypersound modes in $\mathrm{B} 2$ with frequencies $f_{\mathrm{W}} \sim$ $17 \mathrm{GHz}$ remain in the wavepacket at large distances. We shall call these long propagating modes waveguide modes ( $\mathrm{W}$ modes). The reason for this name will become clear after identifying their polarization and spatial distribution. All other modes, including the traditional Rayleigh-like modes in the B1 spectral band (further referred to as R-modes), decay much faster. Figure $3 \mathrm{~b}$ compares the decays of the $\mathrm{R}$ - and $\mathrm{W}$ - modes with distance. Blue circles show the dependence of the spectral amplitude of the first-order Rayleigh mode (R1) at $f_{\mathrm{R} 1}=12.1$ GHz. This mode dominates in the signal measured at $x=0$ and demonstrates the longest propagation among the $\mathrm{R}$ modes. The mean free path for the R1-mode is $\bar{l}_{\mathrm{R} 1}=7.2 \mu \mathrm{m}$, so that it is hardly observable at $x=30 \mu \mathrm{m}$. In contrast, the $\mathrm{W}$ modes have a much larger mean free path $\bar{l}_{\mathrm{W}}$. The distance dependence of their mean spectral amplitude averaged over the B2 frequency band, shown by red squares, demonstrates a nonmonotonic decay and is almost constant at $x>30 \mu \mathrm{m}$. The $\mathrm{W}$-modes are uniquely detected in the time domain at $x=50$ $\mu \mathrm{m}$ and larger (our measurements are limited by the NG length). Figure $3 \mathrm{c}$ shows the filtered signals for the $\mathrm{W}$-modes (B2 spectral band) at several distances from the pump spot. The signals possess beatings, and their exact shape depends on $x$. This indicates that the signal in this spectral band consists of several hypersound modes propagating at different velocities. The inset in Figure $3 c$ shows the linear dependence of the arrival time of the $\mathrm{W}$-modes (determined from the center of the respective wavepackets) on the coordinate $x$. From this dependence, we obtain the mean sound velocity $\bar{s}_{\mathrm{W}}=3170 \mathrm{~m} /$ $\mathrm{s}$, which is slower than the sound velocities for transverse (TA) sound in the $[100]$ direction in metallic $(\mathrm{Fe}, \mathrm{Ga})\left(s_{\mathrm{FeGa}}=3955\right.$ $\mathrm{m} / \mathrm{s})$ and $\mathrm{GaAs}\left(s_{\mathrm{GaAs}}=3346 \mathrm{~m} / \mathrm{s}\right)$, but is essentially faster than the measured speed of sound for the R1-mode in the studied sample $\left(s_{\mathrm{R} 1}=1950 \mathrm{~m} / \mathrm{s}\right)$.

Another nanograting with $d=150 \mathrm{~nm}$ deposited on the GaAs/AlAs SL sublayer shows similar results, but metallic layers deposited onto a GaAs substrate without SL do not show any modes, which propagate on distances larger than 10 $\mu \mathrm{m}$ in any spectral band. The experimental results and the calculated values of sound group velocities $s_{i}$, estimated mean free paths $\bar{l}_{i}$, and maximum distances $l_{i}^{\max }$, at which hypersound is clearly seen in the FFTs above the noise level, are presented in the Supporting Information for all measured samples. Thus, we conclude that the SL subsurface layer is essential to observe hypersound modes propagating on long distances.

To understand the difference in the propagation length for Rayleigh-like and W- hypersound modes, we identify the origins of the observed spectral lines in the FFTs in the phonon dispersion curves. Figure $4 \mathrm{a}$ shows these dispersion curves, $f\left(k_{x}\right)$, for the sample with the $d=200 \mathrm{~nm}$ nanograting in the metallic $\mathrm{Fe}_{0.81} \mathrm{Ga}_{0.19}$ layer, deposited on the GaAs/AlAs SL. Here $k_{x}$ is the Bloch wavevector and $q_{x}=k_{x}+n 2 \pi / d$. Only modes with atom displacements $\mathbf{u}(x, z)$ in the sagittal plane ( $x z$-plane in Figure 1b) are considered. The dispersion curves are shown in the range near the center of the folded Brillouin zone for the frequency range around $n=1$. The calculated dispersions for the plain structure without NG (see Section S2 in Supporting Information) help us to identify the origins of the modes. We attribute the dispersion branches $\mathrm{R} 1$ and $\mathrm{R} 1 *$ to the lowest first-order Rayleigh-like mode. In the periodic structure with NG, this mode is split into the symmetric (R1) and antisymmetric $(\mathrm{R} 1 *)$ modes with even and odd distributions of the $z$-component of the displacement relative to the center of the NG groove, with a spectral gap at $k_{x}=0$. The spatial distributions of these modes are concentrated in the metallic layer, as demonstrated in Figure 4b. Both R1 and $\mathrm{R} 1 *$ modes are observed in the experiments for relatively small distances $2<x<15 \mu \mathrm{m}$ at $f_{\mathrm{R} 1}=12.1 \mathrm{GHz}$ and $f_{\mathrm{R} 1^{*}}=11.2$ $\mathrm{GHz}$, respectively (for example, see the inset in Figure $2 \mathrm{~b}$ ). The modes R2 and R2* are the second-order Rayleigh-like 

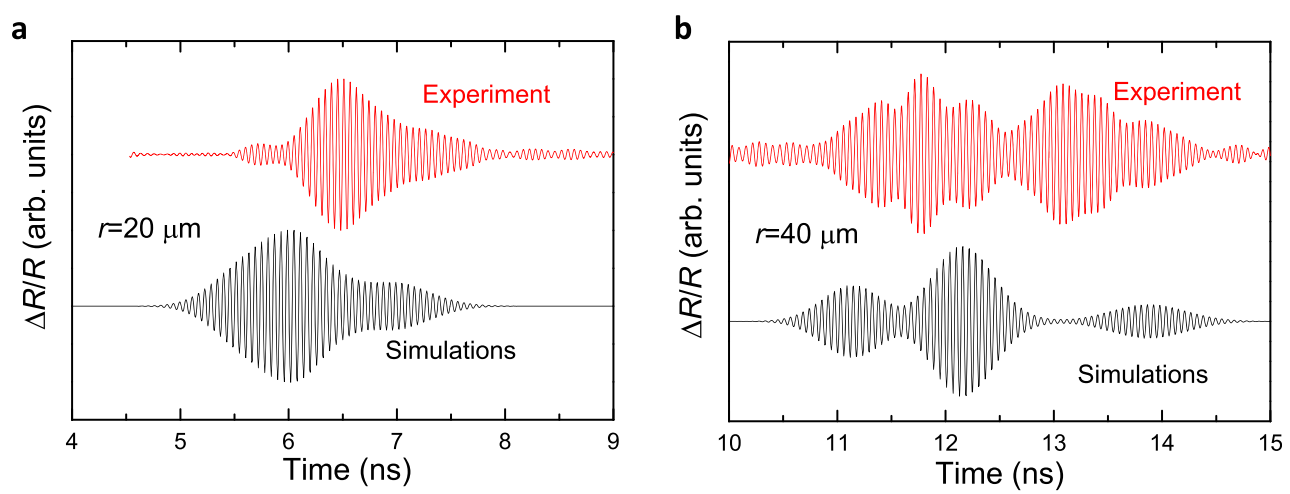

Figure 5. W-mode wavepackets. Measured (red) and simulated (black) phonon wavepackets in the B2 spectral band for two propagation distances: $x=20 \mu \mathrm{m} \mathrm{(a)} \mathrm{and} 40 \mu \mathrm{m}(\mathrm{b})$. In the simulations, four $\mathrm{W}$-modes with frequencies $f_{\mathrm{w}}=16.532,17.026,17.223$, and 17.455 GHz and velocities $s_{\mathrm{w}}=3266,3310,3591$, and $2881 \mathrm{~m} / \mathrm{s}$ are summed.

modes, often called Sezawa-like modes. ${ }^{28}$ Similar to the R1modes, these modes are concentrated in the $(\mathrm{Fe}, \mathrm{Ga})$ layer. The excellent agreement with the calculated frequencies $f_{i}$ and sound velocities $s_{i}$ allows us to identify unambiguously the symmetric ( $\mathrm{R} 1$ and $\mathrm{R} 2$ ) and antisymmetric ( $\mathrm{R}^{*}$ and $\mathrm{R} 2 *$ ) propagating Rayleigh modes in the experimental spectra.

The most important result revealed by the dispersion curves is the existence of the $\mathrm{W}$-modes with linear dispersion branches that are degenerate at $k_{x}=0$ and $f_{\mathrm{W}} \sim 17 \mathrm{GHz}$, that is, with Dirac cone-type dispersions. The striking difference between the $\mathrm{W}$ - and R-modes is their spatial distribution, as shown in Figure $4 \mathrm{~b}$. While the R-modes are concentrated in the metallic layer, the $\mathrm{W}$-modes are localized in the sublayer with the GaAs/AlAs SL, well below the NG. The $\mathrm{W}$-modes have an analogy with the Lamb modes in plates and behave as waveguide modes. In total, there are 11 pairs of $\mathrm{W}$-modes localized in the SL (see Section S3 in Supporting Information for details). Their frequencies and group velocities cover the ranges $f_{\mathrm{W}}=16.5-18 \mathrm{GHz}$ and $s_{\mathrm{W}}=2800-3600 \mathrm{~m} / \mathrm{s}$, respectively. The excellent agreement between calculated and measured frequencies and their velocities leads us to the conclusion that the waveguide $\mathrm{W}$-modes are those with the long propagation lengths in our samples.

The dispersion curves and spatial distributions for the sample without a SL subsurface layer show that R-modes exist there with dispersion relations very similar to the considered case with a SL, but $\mathrm{W}$-modes are not present. This theoretical result confirms that the observation of $\mathrm{W}$-modes is governed exclusively by the presence of the GaAs/AlAs SL which acts as a multichannel waveguide. The same conclusion can be drawn by comparing the signals measured on the samples with and without a SL sublayer.

The exciting property of the $\mathrm{W}$-modes is their ability to propagate parallel to the surface for long distances. This is explained by the localization of the $\mathrm{W}$-modes in the SL layer as shown in Figure 4b. The (Fe,Ga)/SL and SL/GaAs interfaces play the role of confining borders for Lamb-like modes ${ }^{29}$ in the SL sublayer. The structure could be viewed as a waveguiding plate loaded on top by a layer of finite thickness and at the bottom by the infinite substrate. In comparison with a Lamb plate in air or water, the structure is asymmetric both geometrically and in terms of the loading materials. In contrast to Rayleigh waves at a plain surface, the corrugation results in the leakage of both $\mathrm{R}$ - and $\mathrm{W}$-modes to the bulk. ${ }^{18}$ However, the calculations show that the losses into the bulk modes are much smaller for the guided $\mathrm{W}$-modes than for the R-modes.
The calculated quality factor, $Q$ reaches $\sim 10^{5}$ for the $\mathrm{W}$ modes, but does not exceed $\sim 10^{3}$ for the R-modes (see Table S2 in the Supporting Information). This difference is explicitly demonstrated in the Supporting Video 1, which animates the calculated propagation of the excited hypersound in the studied sample and visualizes the emissions from R-modes and $\mathrm{W}$-modes into bulk waves. When the $\mathrm{R}$-modes and $\mathrm{W}$-modes are sufficiently spatially separated to distinguish these two emissions in the animation (after 4.5 ns delay from the optical excitation), the strong leakage of R-modes to the bulk is clearly observed, while the weak leakage of $\mathrm{W}$-modes is hardly visible. The linear dispersion of $\mathrm{W}$-modes (see Figure 4a), in addition to their high $Q$ factors, also indicates that the grating, which causes the excitation of the hypersound waveguide modes, has a small influence on their propagation. Thus, the much larger broadening of the R-modes' wavepacket in comparison with the wavepacket of the $\mathrm{W}$-modes is clearly visible already at small $x$ (compare the filtered signals for the B1 and B2 bands in Figure 2b).

The waveguiding observed experimentally is a multimodal effect. The bunch of propagating $\mathrm{W}$-modes covers the frequency range from 16 to $18 \mathrm{GHz}$ (see the FFTs in Figure $3 a)$. The temporal signals and their FFTs allow us to distinguish several modes propagating with different velocities. As a result, we observe temporal beatings in the signals presented in Figure $3 \mathrm{c}$ and a nonmonotonic dependence of the mean spectral density on the coordinate $x$ in Figure $3 \mathrm{~b}$. To illustrate the multimodal character of the waveguiding effect, we present a simulation of the transient reflectivity signal induced by a propagating multimode wavepacket in the Supporting Video 2. Two signals from this simulation are presented together with the experimental signals for $x=20$ and $40 \mu \mathrm{m}$ in Figure 5. In the simulations, we did not aim for a perfect agreement with the experiment and limited the signal in the $\mathrm{B} 2$ band to the sum of four $\mathrm{W}$-modes that are simultaneously excited at the excitation spot with the same amplitudes, spatial distributions, and initial phases. Their frequencies $f_{\mathrm{w}}=16.532,17.026,17.223$, and $17.455 \mathrm{GHz}$ and corresponding velocities $s_{\mathrm{w}}=3266,3310,3591$, and $2881 \mathrm{~m} / \mathrm{s}$ are taken from the calculated dispersion curves in Figure $4 \mathrm{a}$ and correspond to $k_{x}=0.01 \pi / d$. It is seen that the arrival time of the hypersound bunch and its temporal width are in good agreement with the experiment. The simulated spatialtemporal evolution of the signal shows also pronounced beatings, which agrees with the experimental observations and 
supports the conclusion about the multimodal character of the waveguiding effect.

\section{CONCLUSIONS}

In summary, we have realized experimentally the generation and detection of acoustic waves with a frequency up to 20 $\mathrm{GHz}$, which propagate underneath a corrugated surface. These hypersonic waves propagate on a distance of more than $50 \mu \mathrm{m}$ and lose their energy to bulk phonons much less efficiently than Rayleigh waves, which propagate only on several micrometers due to scattering on the nanostructured surfaces.

For prospective applications in communications, it is important that the subsurface $\mathrm{W}$-modes faithfully carry the surface morphology imprinted at the point of excitation, which must then be read-out at the point of detection. In the particular case described in the present experiments, the morphology is carried by the generated and detected hypersound wavevector $q_{x} \approx 2 \pi / d$, defined by the NG period. In the general case, the excitation and detection points could include nanophononic cavities ${ }^{30}$ coupled with the subsurface waveguide. A further advantage over traditional Rayleigh-like modes may be taken from the bunched character of the subsurface $\mathrm{W}$-modes, the number of which is controlled by the width of the waveguiding layer. This makes possible using the same subsurface waveguide for encoding information at different acoustic frequencies with the same $q_{x}$ by resonant driving. ${ }^{31}$

The possibility to deliver information by hypersound well beneath corrugated surfaces on macroscopic distances has a direct perspective to control a single photon emission from buried quantum nanoobjects, ${ }^{8,32,33}$ which quantum efficiency decreases strongly when located near the surface. Subsurface hypersound may be used to control the emission from a Bose condensate in polariton microcavities located at a micrometer distance from the surface. ${ }^{34}$ The technique may be applied to control exciton resonances in two-dimensional van der Waals nanolayers, which require passivation for high quantum efficiency. ${ }^{35,36}$ The $(\mathrm{Fe}, \mathrm{Ga})$ metallic layer used in our experiment is a ferromagnet, known as Galfenol. ${ }^{37}$ It possesses a strong magnon-phonon coupling which allows the conversion of magnon excitations to hypersound and vice versa. $^{38}$ In magnonic devices, the described concept of a nanostructured ferromagnetic layer deposited on a hypersound waveguide can be used for transmitting coherent spin excitations. $^{39,40}$ The preliminary measurements show that despite the dominating localization of the $\mathrm{W}$-modes in the SL, their tail located in the Galfenol layer has a sufficiently large amplitude for carrying coherent magnons of the corresponding frequencies for distances exceeding $50 \mu \mathrm{m}$. This effect, however, is out of the scope of the present paper and will be addressed elsewhere.

It is appealing to excite the guided subsurface modes electrically without using bulky ultrafast lasers. This method, however, should be different from standard techniques for generating SAWs with interdigital transducers. It is necessary to excite a bulk hypersonic wave, which propagates perpendicular to the surface and carries the information encoded at the surface (e.g. by a grating) in terms of its lateral amplitude and phase modulation. When incident on the undersurface waveguide, this laterally modulated wave transfers the encoded information to the $\mathrm{W}$-modes. The electrical generation of bulk hypersonic waves at frequencies higher than $10 \mathrm{GHz}$ is challenging. Recently such generation of hyper- sound with frequency up to $20 \mathrm{GHz}$ has been demonstrated with piezoelectric $\mathrm{ZnO}$ transducers; ${ }^{41}$ application of such an electrical technique to the corrugated surface could lead to wider applications of subsurface hypersound.

\section{METHODS}

Sample Production. The studied samples were epitaxially grown on commercial GaAs substrates [(100)-semi-insulating GaAs]. The SL was produced by molecular beam epitaxy, and the $\mathrm{Fe}_{0.81} \mathrm{Ga}_{0.19}$ layers were deposited by magnetron sputtering. The nanogratings were fabricated through focused ion beam milling (Raith VELION) with both 100 and $75 \mathrm{~nm}$ lines and spaces over an area of $25 \mu \mathrm{m} \times$ $100 \mu \mathrm{m}$. To ensure high beam resolution during the patterning of the gratings, a low $\mathrm{Ga}^{+}$beam current of $22 \mathrm{pA}$ was applied at $35 \mathrm{keV}$ beam energy. The milling dose was set to $0.3 \mathrm{nC} / \mu \mathrm{m}^{2}$, resulting in the groove depth of $25 \mathrm{~nm}$.

Time-Resolved Pump-Probe Measurements. The pumpprobe scheme was realized using two mode-locked erbium-doped ring fiber lasers (TOPTICA FemtoFiber Ultra 1050 and FemtoFiber Ultra 780). The lasers generate pulses of $150 \mathrm{fs}$ duration with a repetition rate of $80 \mathrm{MHz}$ at wavelengths of $1050 \mathrm{~nm}$ (pump pulses) and 780 $\mathrm{nm}$ (probe pulses). The pump beam was focused by a microscope objective $(20 \times ; \mathrm{NA}=0.4)$ to the backside of the metal layer through the GaAs substrate and GaAs/AlAs SL, which are transparent for the pump wavelength. The focused pump spot had a Gaussian intensity distribution $\sim \exp \left(-\frac{r^{2}}{2 \sigma^{2}}\right)$, where $r$ is the distance from the center of the spot and $\sigma=1 \mu \mathrm{m}$ is the spot radius at $1 / \sqrt{ } e$ level. The maximal used pulsed excitation density was $8 \mathrm{~mJ} / \mathrm{cm}^{2}$. The probe beam was focused by another microscope objective $(100 \times$; NA $=0.8)$ to the front side of the sample. The focused probe spot had a Gaussian distribution also with $\sigma=0.5 \mu \mathrm{m}$ and an energy density of $2 \mathrm{~mJ} / \mathrm{cm}^{2}$. The temporal resolution was achieved employing an asynchronous optical sampling technique. ${ }^{42}$ The pump and probe oscillators were locked with a frequency offset of $1600 \mathrm{~Hz}$. In combination with the 80 $\mathrm{MHz}$ repetition rate, it allowed measurement of the time-resolved signals in a time window of $12.5 \mathrm{~ns}$ with a time resolution of $<1 \mathrm{ps}$. The distance between the centers of the pump and probe spots was controlled with a precision of $0.05 \mu \mathrm{m}$. Two-dimensional spatial scanning across the nanograting surface confirmed the directional propagation of the hypersound wavepackets along the reciprocal lattice vector of the nanograting. At the considered propagation distances, the influence of diffraction on these acoustic beams is negligible and cannot be observed. The measured size of the hypersound beams and the $y$-distribution of the modes' amplitudes correspond to the size of the pump spot at the point of excitation. Only the low-frequency background Rayleigh wave [see the filtered $(<5 \mathrm{GHz})$ signal in Figure $2 \mathrm{~b}]$ is emitted in all surface directions and exhibits cylindrical symmetry.

Transformation of Optically Generated Stress to Hypersound. The guided modes of interest belong to the system of acoustic eigenmodes of the sample, which are all spatially periodic because of the periodicity imposed by the nanograting (FloquetBloch theorem). Each eigenmode contains an infinite number of wavevectors: $q_{x}=k_{x}+n(2 \pi / d)$, where $k_{x}$ is the Bloch vector, $d$ is the NG period, and $n$ is an integer, but one spatial harmonic with a certain $n$ dominates. A particular acoustic eigenmode is excited with nonzero amplitude when the frequency spectrum of the photoinduced stress contains the frequency of the eigenmode and the lateral distribution of the stress contains the wavevector of this mode. ${ }^{22}$ The intensity envelope of the fs laser pulses applied in our experiments contains frequencies up to $\sim 0.1 \mathrm{THz}$ and, thus, covers the frequencies of the considered guided modes. In contrast, the spatial spectrum of the stress that is induced by the pump light focused on the interface of the SL and metallic layer (opposite to the open surface with the NG) is limited by the spatial pump intensity distribution. For a Gaussian distribution of the laser intensity along the $x$-axis $\sim \exp \left(-\frac{x^{2}}{2 \sigma^{2}}\right)$, the spectrum of the wavevectors, $q_{\mathrm{P}}$, generated by the photoexcited stress 
is proportional to $\exp \left(-\frac{q_{\mathrm{P}}^{2} \sigma^{2}}{2}\right)$. Due to the large size of the laser spot in comparison with the NG period, this spectrum does not contain components of $q_{x}$ with nonzero $n$. However, the generation of all acoustic eigenmodes is possible due to the matching of the photoinduced stress and the $n=0$ components of these eigenmodes.

Calculation of Hypersound Dispersion and Spatial Distribution of Phonon Modes. We use COMSOL Multiphysics for calculation of the phonon dispersion in our system. For this, we consider a NG unit cell and apply Floquet-Bloch periodic boundary conditions, that is, $u_{\mathrm{d}}=u_{\mathrm{s}} \exp \left(-i k_{x} d\right)$, where $u_{\mathrm{d}}$ and $u_{\mathrm{s}}$ are the atom displacements at the destination and source boundaries, respectively, and $k_{x}$ is a Bloch wavenumber. At the open NG surface, we use free boundary conditions. For the simulation of the semi-infinite substrate, we use a perfectly matched layer (PML) at the backside of the substrate. The stiffness tensor components, $C_{k l}$, and the mass density, $\rho$, used for the calculations are for $\mathrm{Fe}_{0.81} \mathrm{Ga}_{0.19}$ (ref 32): $C_{11}=209$ $\mathrm{GPa}, C_{12}=156 \mathrm{GPa}, C_{44}=122 \mathrm{GPa}, \rho=7800 \mathrm{~kg} / \mathrm{m}^{3}$; for $\mathrm{GaAs} C_{11}=$ $119 \mathrm{GPa}, C_{12}=53.8 \mathrm{GPa}, C_{44}=59.5 \mathrm{GPa}, \rho=5316 \mathrm{~kg} / \mathrm{m}^{3}$; for AlAs $C_{11}=119.9 \mathrm{GPa}, C_{12}=57.5 \mathrm{GPa}, C_{44}=56.6 \mathrm{GPa}, \rho=3760 \mathrm{~kg} / \mathrm{m}^{3}$. The dispersion and modes' spatial distributions are obtained by an eigenfrequency analysis. Most of the solutions are shown in Figure 4a. There are also solutions arising due to the finite size of the simulated substrate, but they are not related to the experimentally observed effects and are, thus, ignored. To select the modes localized in the NG and SL, we used the approach described elsewhere. ${ }^{43}$ We characterize the localization degree with the quality factor $Q$ and parameter $p$ defined by

$$
p=\frac{\left\langle F_{\mathrm{e}}\right\rangle_{\mathrm{L}}}{\left\langle F_{\mathrm{e}}\right\rangle_{\mathrm{S}}}
$$

where $F_{\mathrm{e}}$ is the elastic free energy density and the averaging is performed over two areas: The L-area includes the $\mathrm{Fe}_{0.81} \mathrm{Ga}_{0.19}$ film and the SL, and the S-area corresponds to the PML. Only W-modes with $p \gg 1$ propagate for a long distance. The value of $p$ depends on $q_{x}$. The calculations show that for the $\mathrm{Fe}_{0.81} \mathrm{Ga}_{0.19}$ film $p>100$ and $Q$ $>5 \times 10^{3}$ for $4 \mathrm{~W}$-modes at $q_{x} \approx 2 \pi / d$ (see Section S3 in Supporting Information). These four $\mathrm{W}$-modes from the total of 11 pairs are considered as the best candidates for long propagating subsurface modes in our experiments. The existence of the $\mathrm{W}$-modes with long propagation length is confirmed by a semianalytical consideration which is described in Supporting Information (Section S2).

\section{ASSOCIATED CONTENT}

\section{SI Supporting Information}

The Supporting Information is available free of charge at https://pubs.acs.org/doi/10.1021/acsnano.0c09475.

Experimental and theoretical data for all the studied samples; elastic equations and their semi-analytical solutions for an unpatterned metallic film on a GaAs substrate and on a SL; spatial distributions and main parameters of the $\mathrm{W}$-modes for the grating with $200 \mathrm{~nm}$ period (PDF)

Video 1: Spatial-temporal modeling (COMSOL Multiphysics) of the hypersound propagation in the structure with a GaAs/AlAs SL and a $\mathrm{Fe}_{0.81} \mathrm{Ga}_{0.19}$ nanograting with period $d=200 \mathrm{~nm}$ after excitation by the pump pulse. The color map illustrates the time derivative of the $z$ component of the displacement vector. The black horizontal lines show the boundaries between the different materials of the structure (MOV)

Video 2: Temporal evolution of the guided wavepacket consisting of four $\mathrm{W}$-modes with frequencies $f_{\mathrm{w}}=16.532$, $17.026,17.223$, and $17.455 \mathrm{GHz}$ and corresponding velocities $s_{\mathrm{w}}=3266,3310,3591$, and $2881 \mathrm{~m} / \mathrm{s}$, taken from the calculated dispersions. The modes are simultaneously excited at the excitation spot with the same amplitudes, spatial distributions, and initial phases (MOV)

\section{AUTHOR INFORMATION}

\section{Corresponding Author}

Alexey V. Scherbakov - Experimentelle Physik 2, Technische Universität Dortmund, 44227 Dortmund, Germany; Ioffe Institute, 194021 St. Petersburg, Russia; 이이.org/00000001-7358-4164; Email: alexey.shcherbakov@tudortmund.de

\section{Authors}

Dmytro D. Yaremkevich - Experimentelle Physik 2, Technische Universität Dortmund, 44227 Dortmund, Germany

Serhii M. Kukhtaruk - Experimentelle Physik 2, Technische Universität Dortmund, 44227 Dortmund, Germany; Department of Theoretical Physics, V. E. Lashkaryov Institute of Semiconductor Physics, 03028 Kyiv, Ukraine

Tetiana L. Linnik - Department of Theoretical Physics, V. E. Lashkaryov Institute of Semiconductor Physics, 03028 Kyiv, Ukraine

Nikolay E. Khokhlov - Ioffe Institute, 194021 St. Petersburg, Russia

Felix Godejohann - Experimentelle Physik 2, Technische Universität Dortmund, 44227 Dortmund, Germany; (1) orcid.org/0000-0001-7237-1650

Olga A. Dyatlova - Experimentelle Physik 2, Technische Universität Dortmund, 44227 Dortmund, Germany

Achim Nadzeyka - Raith GmbH, 44263 Dortmund, Germany

Debi P. Pattnaik - School of Physics and Astronomy, University of Nottingham, Nottingham NG7 2RD, United Kingdom

Mu Wang - School of Physics and Astronomy, University of Nottingham, Nottingham NG7 2RD, United Kingdom

Syamashree Roy - School of Physics and Astronomy, University of Nottingham, Nottingham NG7 2RD, United Kingdom

Richard P. Campion - School of Physics and Astronomy, University of Nottingham, Nottingham NG7 2RD, United Kingdom

Andrew W. Rushforth - School of Physics and Astronomy, University of Nottingham, Nottingham NG7 2RD, United

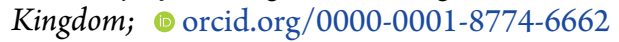

Vitalyi E. Gusev - LAUM, CNRS UMR 6613, Le Mans Université, 72085 Le Mans, France

Andrey V. Akimov - School of Physics and Astronomy, University of Nottingham, Nottingham NG7 2RD, United Kingdom; () orcid.org/0000-0002-8173-8212

Manfred Bayer - Experimentelle Physik 2, Technische Universität Dortmund, 44227 Dortmund, Germany; Ioffe Institute, 194021 St. Petersburg, Russia; (1) orcid.org/00000002-0893-5949

Complete contact information is available at: https://pubs.acs.org/10.1021/acsnano.0c09475

\section{Author Contributions}

D.Y. and F.G. constructed the experimental setup. D.Y, N.E.K, F.G., and O.A.D carried out the experiment and performed the data analysis. A.V.S. and A.V.A. supervised the research. 
S.M.K., T.L.L., and V.E.G. performed theoretical analysis and numerical modeling. A.N. produced and characterized the nanogratings. D.P.P., M.W., S.R, R.P.C, and A.W.R. grew and characterized the samples. A.V.S., S.M.K., T.L.L., A.W.R., V.E.G., A.V.A., and M.B. discussed the results and wrote the manuscript.

\section{Notes}

The authors declare no competing financial interest.

\section{ACKNOWLEDGMENTS}

The work was supported by the Bundesministerium fur Bildung und Forschung through the project VIP+ "Nanomagnetron" and by the Deutsche Forschungsgemeinschaft in the frame of the Collaborative Research Center TRR 142 (project A06 "Tailored ultrafast acoustics for light emission modulation"). The cooperation between TU Dortmund, the Lashkaryov Institute, and the Ioffe Institute was supported by the Volkswagen Foundation (grant no. 97758).

\section{REFERENCES}

(1) Satzinger, K. J.; Zhong, Y. P.; Chang, H.-S.; Peairs, G. A.; Bienfait, A.; Chou, M.-H.; Cleland, A. Y.; Conner, C. R.; Dumur, É.; Grebel, J.; Gutierrez, I.; November, B. H.; Povey, R. G.; Whiteley, S. J.; Awschalom, D. D.; Schuster, D. I.; Cleland, A. N. Quantum Control of Surface Acoustic-Wave Phonons. Nature 2018, 563, 661665.

(2) Delsing, P.; Cleland, A. N.; Schuetz, M. J. A.; Knörzer, J.; Giedke, G.; Cirac, J. I.; Srinivasan, K.; Wu, M.; Balram, K. C.; Bäuerle, C.; Meunier, T.; Ford, C. J. B.; Santos, P. V.; Cerda-Méndez, E.; Wang, H.; Krenner, H. J.; Nysten, E. D. S.; Weiß, M.; Nash, G. R.; Thevenard, L.; et al. The 2019 Surface Acoustic Waves Roadmap. J. Phys. D: Appl. Phys. 2019, 52, 353001.

(3) Hann, C. T.; Zou, C.-L.; Zhang, Y.; Chu, Y.; Schoelkopf, R. J.; Girvin, S. M.; Jiang, L. Hardware-Efficient Quantum Random Access Memory with Hybrid Quantum Acoustic Systems. Phys. Rev. Lett. 2019, 123, 250501.

(4) Clerk, A. A.; Lehnert, K. W.; Bertet, P.; Petta, J. R.; Nakamura, Y. Hybrid Quantum Systems with Circuit Quantum Electrodynamics. Nat. Phys. 2020, 16, 257-267.

(5) Bertrand, B.; Hermelin, S.; Takada, S.; Yamamoto, M.; Tarucha, S.; Ludwig, A.; Wieck, A. D.; Bäuerle, C.; Meunier, T. Fast Spin Information Transfer Between Distant Quantum Dots Using Individual Electrons. Nat. Nanotechnol. 2016, 11, 672-676.

(6) Takada, S.; Edlbauer, H.; Lepage, H. V.; Wang, J.; Mortemousque, P.-A.; Georgiou, G.; Barnes, C. H. W.; Ford, C. J. B.; Yuan, M. Y.; Santos, P. V.; Waintal, X.; Ludwig, A.; Wieck, A. D.; Urdampilleta, M.; Meunier, T.; Bäuerle, C. Sound-Driven SingleElectron Transfer in a Circuit of Coupled Quantum Rails. Nat. Commun. 2019, 10, 4557.

(7) Vogele, A.; Sonner, M. M.; Mayer, B.; Yuan, X.; Weiß, M.; Nysten, E. D. S.; Covre da Silva, S. F.; Rastelli, A.; Krenner, H. J. Quantum Dot Optomechanics in Suspended Nanophononic Strings. Adv. Quantum Technol. 2020, 3, 1900102.

(8) Hsiao, T.-K.; Rubino, A.; Chung, Y.; Son, S.-K.; Hou, H.; Pedrós, J.; Nasir, A.; Éthier-Majcher, G.; Stanley, M. J.; Phillips, R. T.; Mitchell, T. A.; Griffiths, J. P.; Farrer, I.; Ritchie, D. A.; Ford, C. J. B. Single-Photon Emission from Single-Electron Transport in a SAWDriven Lateral Light-Emitting Diode. Nat. Commun. 2020, 11, 917.

(9) Golter, D. A.; Oo, T.; Amezcua, M.; Stewart, K. A.; Wang, H. Optomechanical Quantum Control of a Nitrogen-Vacancy Center in Diamond. Phys. Rev. Lett. 2016, 116, 143602.

(10) Labanowski, D.; Bhallamudi, V. P.; Guo, Q.; Purser, C. M.; McCullian, M. A.; Hammel, P. C.; Salahuddin, S. Voltage-Driven, Local, and Efficient Excitation of Nitrogen-Vacancy Centers in Diamond. Sci. Adv. 2018, 4, eaat6574.

(11) Maity, S.; Shao, L.; Bogdanović, S.; Meesala, S.; Sohn, Y.-I.; Sinclair, N.; Pingault, B.; Chalupnik, M.; Chia, C.; Zheng, L.; Lai, K.;
Lončar, M. Coherent Acoustic Control of a Single Silicon Vacancy Spin in Diamond. Nat. Commun. 2020, 11, 193.

(12) Gustafsson, M. V.; Aref, T.; Kockum, A. F.; Ekstrom, M. K.; Johansson, G.; Delsing, P. Propagating Phonons Coupled to an Artificial Atom. Science 2014, 346, 207-211.

(13) Lemonde, M.-A.; Meesala, S.; Sipahigil, A.; Schuetz, M. J. A.; Lukin, M. D.; Loncar, M.; Rabl, P. Phonon Networks with SiliconVacancy Centers in Diamond Waveguides. Phys. Rev. Lett. 2018, 120, 213603.

(14) Bienfait, A.; Satzinger, K. J.; Zhong, Y. P.; Chang, H.-S.; Chou, M.-H.; Conner, C. R.; Dumur, E.; Grebel, J.; Peairs, G. A.; Povey, R. G.; Cleland, A. N. Phonon-Mediated Quantum State Transfer and Remote Qubit Entanglement. Science 2019, 364, 368-371.

(15) Devaux, T.; Tozawa, H.; Otsuka, P. H.; Mezil, S.; Tomoda, M.; Matsuda, O.; Bok, E.; Lee, S. H.; Wright, O. B. Giant Extraordinary Transmission of Acoustic Waves Through a Nanowire. Sci. Adv. 2020, 6 , eaay8507.

(16) Kukushkin, I. V.; Smet, J. H.; Scarola, V. W.; Umansky, V.; von Klitzing, K. Dispersion of the Excitations of Fractional Quantum Hall States. Science 2009, 324, 1044-1047.

(17) Schubert, M.; Grossmann, M.; Ristow, O.; Hettich, M.; Bruchhausen, A.; Barretto, E. C. S.; Scheer, E.; Gusev, V.; Dekorsy, T. Spatial-Temporally Resolved High-Frequency Surface Acoustic Waves on Silicon Investigated by Femtosecond Spectroscopy. Appl. Phys. Lett. 2012, 101, 013108.

(18) Glass, N. E.; Maradudin, A. A. Leaky Surface Elastic Waves on Both Flat and Strongly Corrugated Surfaces for Isotropic, Nondissipative Media. J. Appl. Phys. 1983, 54, 796-805.

(19) Urick, R. J. Principles of Underwater Sound; 3rd ed.; McGrawHill: New York, 1983.

(20) Thomsen, C.; Grahn, H. T.; Maris, H. J.; Tauc, J. Surface Generation and Detection of Phonons by Picosecond Light Pulses. Phys. Rev. B: Condens. Matter Mater. Phys. 1986, 34, 4129-4138.

(21) Matsuda, O.; Larciprete, M. C.; Li Voti, R.; Wright, O. B. Fundamentals of Picosecond Laser. Ultrasonics 2015, 56, 3-20.

(22) Hurley, D. H.; Telschow, K. L. Picosecond Surface Acoustic Waves Using a Suboptical Wavelength Absorption Grating. Phys. Rev. B: Condens. Matter Mater. Phys. 2002, 66 (2002), 153301.

(23) Giannetti, C.; Revaz, B.; Banfi, F.; Montagnese, M.; Ferrini, G.; Cilento, F.; Maccalli, S.; Vavassori, P.; Oliviero, G.; Bontempi, E.; Depero, L. E.; Metlushko, V.; Parmigiani, F. Thermomechanical Behavior of Surface Acoustic Waves in Ordered Arrays of Nanodisks Studied by Near-Infrared Pump-Probe Diffraction Experiments. Phys. Rev. B: Condens. Matter Mater. Phys. 2007, 76, 125413.

(24) Sadhu, J.; Lee, J. H.; Sinha, S. Frequency Shift and Attenuation of Hypersonic Surface Acoustic Phonons under Metallic Gratings. Appl. Phys. Lett. 2010, 97, 133106.

(25) Maznev, A. A.; Wright, O. B. Optical Generation of Long-Lived Surface Vibrations in a Periodic Microstructure. J. Appl. Phys. 2009, $105,123530$.

(26) Bjornsson, M. M.; Connolly, A. B.; Mahat, S.; Rachmilowitz, B. E.; Daly, B. C.; Antonelli, G. A.; Myers, A.; Singh, K. J.; Yoo, H. J.; King, S. W. Picosecond Ultrasonic Study of Surface Acoustic Waves on Titanium Nitride Nanostructures. J. Appl. Phys. 2015, 117, 095305.

(27) Sugawara, Y.; Wright, O. B.; Matsuda, O.; Takigahira, M.; Tanaka, Y.; Tamura, S.; Gusev, V. E. Watching Ripples on Crystals. Phys. Rev. Lett. 2002, 88, 185504.

(28) Farnell, G. W.; Adler, E. L. Elastic Wave Propagation in Thin Layers; Physical Acoustics: Principles and Methods; Mason, W. P., Thurston, R. N., Eds.; Academic Press: New York, 1972; Vol. 9; pp 35-127.

(29) Grossmann, M.; Ristow, O.; Hettich, M.; He, C.; Waitz, R.; Scheer, E.; Gusev, V.; Dekorsy, T.; Schubert, M. Time-Resolved Detection of Propagating Lamb Waves in Thin Silicon Membranes with Frequencies up to $197 \mathrm{GHz}$. Appl. Phys. Lett. 2015, 106, 171904.

(30) Shao, L.; Maity, S.; Zheng, L.; Wu, L.; Shams-Ansari, A.; Sohn, Y.-I.; Puma, E.; Gadalla, M. N.; Zhang, M.; Wang, C.; Hu, E.; Lai, K.; Lončar, M. Phononic Band Structure Engineering for High-Q 
Gigahertz Surface Acoustic Wave Resonators on Lithium Niobate. Phys. Rev. Appl. 2019, 12, 014022.

(31) Jäckl, M.; Belotelov, V. I.; Akimov, I. A.; Savochkin, I. V.; Yakovlev, D. R.; Zvezdin, A. K.; Bayer, M. Magnon Accumulation by Clocked Laser Excitation as Source of Long-Range Spin Waves in Transparent Magnetic Films. Phys. Rev. X 2017, 7, 021009.

(32) Couto, O. D. D., Jr.; Lazic, S.; Iikawa, F.; Stotz, J. A. H.; Jahn, U.; Hey, R.; Santos, P. V. Photon Anti-Bunching in Acoustically Pumped Quantum Dots. Nat. Photonics 2009, 3, 645-648.

(33) Weiß, M.; Kapfinger, S.; Reichert, T.; Finley, J. J.; Wixforth, A.; Kaniber, M.; Krenner, H. J. Surface Acoustic Wave Regulated Single Photon Emission from a Coupled Quantum Dot-Nanocavity System. Appl. Phys. Lett. 2016, 109, 033105.

(34) Cerda-Mendez, E. A.; Krizhanovskii, D. N.; Wouters, M.; Bradley, R.; Biermann, K.; Guda, K.; Hey, R.; Santos, P. V.; Sarkar, D.; Skolnick, M. S. Polariton Condensation in Dynamic Acoustic Lattices. Phys. Rev. Lett. 2010, 105, 116402.

(35) Alexeev, E. M.; Ruiz-Tijerina, D. A.; Danovich, M.; Hamer, M. J.; Terry, D. J.; Nayak, P. K.; Ahn, S.; Pak, S.; Lee, J.; Sohn, J. I.; Molas, M. R.; Koperski, M.; Watanabe, K.; Taniguchi, T.; Novoselov, K. S.; Gorbachev, R. V.; Shin, H. S.; Fal'ko, V. I.; Tartakovskii, A. I. Resonantly Hybridized Excitons in Moire Superlattices in van der Waals Heterostructures. Nature 2019, 567, 81-86.

(36) Kang, S.; Kim, K.; Kim, B. H.; Kim, J.; Sim, K. I.; Lee, J. U.; Lee, S.; Park, K.; Yun, S.; Kim, T.; Nag, A.; Walters, A.; GarciaFernandez, M.; Li, J.; Chapon, L.; Zhou, K. J.; Son, Y. W.; Kim, J. H.; Cheong, H.; Park, J. G. Coherent Many-Body Exciton in van der Waals Antiferromagnet NiPS3. Nature 2020, 583, 785-789.

(37) Clark, A. E.; Hathaway, K. B.; Wun-Fogle, M.; Restorff, J. B.; Lograsso, T. A.; Keppens, V. M.; Petculescu, G.; Taylor, R. A. Extraordinary Magnetoelasticity and Lattice Softening in bcc Fe-Ga Alloys. J. Appl. Phys. 2003, 93, 8621-8623.

(38) Godejohann, F.; Scherbakov, A. V.; Kukhtaruk, S. M.; Poddubny, A. N.; Yaremkevich, A. N.; Wang, M.; Nadzeyka, A.; Yakovlev, D. R.; Rushforth, A. W.; Akimov, A. V.; Bayer, M. Magnon Polaron Formed by Selectively Coupled Coherent Magnon and Phonon Modes of a Surface Patterned Ferromagnet. Phys. Rev. B: Condens. Matter Mater. Phys. 2020, 102, 144438.

(39) Casals, B.; Statuto, N.; Foerster, M.; Hernández-Mínguez, A.; Cichelero, R.; Manshausen, P.; Mandziak, A.; Aballe, L.; Hernàndez, J. M.; Macià, F. Generation and Imaging of Magnetoacoustic Waves over Millimeter Distances. Phys. Rev. Lett. 2020, 124, 137202.

(40) An, K.; Litvinenko, A. N.; Kohno, R.; Fuad, A. A.; Naletov, V. V.; Vila, L.; Ebels, U.; de Loubens, G.; Hurdequint, H.; Beaulieu, N.; Ben Youssef, J.; Vukadinovic, N.; Bauer, G. E. W.; Slavin, A. N.; Tiberkevich, V. S.; Klein, O. Coherent Long-Range Transfer of Angular Momentum Between Magnon Kittel Modes by Phonons. Phys. Rev. B: Condens. Matter Mater. Phys. 2020, 101, 060407.

(41) Machado, D. H.; Crespo-Poveda, A.; Kuznetsov, A. S.; Biermann, K.; Scalvi, L. V.; Santos, P. V. Generation and Propagation of Superhigh-Frequency Bulk Acoustic Waves in GaAs. Phys. Rev. Appl. 2019, 12, 044013.

(42) Bartels, A.; Cerna, R.; Kistner, C.; Thoma, A.; Hudert, F.; Janke, C.; Dekorsy, T. Ultrafast Time-Domain Spectroscopy Based on High-Speed Asynchronous Optical Sampling. Rev. Sci. Instrum. 2007, $78,035107$.

(43) Graczykowski, B.; Mielcarek, S.; Trzaskowska, A.; Sarkar, J.; Hakonen, P.; Mroz, B. Tuning of a Hypersonic Surface Phononic Band Gap Using a Nanoscale Two-Dimensional Lattice of Pillars. Phys. Rev. B: Condens. Matter Mater. Phys. 2012, 86, 085426. 